\title{
The filled-duration illusion: The function of temporal and nontemporal set
}

\author{
LOUIS M. GOMEZ and LYNN C. ROBERTSON \\ University of California, Berkeley, California 94720
}

\begin{abstract}
Studies of the perception of time have demonstrated that large visual stimuli presented for the same duration as small stimuli will appear to have been presented longer. Two theoretical approaches have been offered to explain this effect. Both have emphasized the absolute amount of information in the stimulus. Thus, both would predict the effect when size is varied between sessions. The present study found no effect of size when size was held constant within sessions, but a substantial effect resulted when size was varied within sessions. Also examined was the range of stimulus durations used within a session. In one condition, patterns of various sizes were presented at 15,30 , or $45 \mathrm{msec}$, while in a second condition, the same patterns were presented to a different group of subjects at 15,85 , or $155 \mathrm{msec}$. The size effect was significantly reduced when longer time intervals were presented. A third group of subjects was shown patterns at even longer times but with a reduced range $(155,170$, and $185 \mathrm{msec})$. Here the size effect was of equal magnitude to that of the 15 msec interval condition. The implications of these results are that current theoretical explanations of the filled-duration illusion rely on incorrect assumptions.
\end{abstract}

Theoretical explanations of the ability to process duration information fall into two general classes. First, there are theories which account for duration discrimination by appealing to the idea of a direct time estimation process. Such a process would, for example, count the number of sensory pulses that occur in any given interval. A comparison process would then measure the output of the counter for two or more intervals resulting in temporal discrimination performance. All direct duration-estimation mechanisms in this approach have one fact in common. They were designed to work on empty intervals (e.g., darkness bounded by flashes of light) or intervals filled with simple homogeneous stimulus items (e.g., pure tones) (Allan \& Kristofferson, 1974).

The second class of theory addresses itself to duration perception in more complex situations. The additional complexity is usually brought about by filling the to-be-judged interval with heterogeneous stimulus items. The stimuli that fill the interval can range from irregular auditory messages to visually presented words of different frequencies (Avant, Lyman, \& Antes, 1975; Ornstein, 1969; Warm \& McCray, 1969). The addition of complex stimuli to the interval makes

This work was partially supported by USPHS Grant NS07787 to Ervin Hafter and by USPHS Training Grant GM1207 to the present authors through the Institute of Human Learning. We are happy to thank E.R. Hafter, C. D. Hardyck, E. A. C. Thomas, T. D. Wickens, and an anonymous reviewer for comments that greatly improved the quality of this manuscript. Reprint requests should be sent to either author at the Institute of Human Learning, University of California, Berkeley, California 94720. the apparent duration longer when compared to a blank interval of the same duration (Buffardi, 1971; Thomas \& Brown, 1974; Thomas \& Weaver, 1975). This phenomenon has been termed the filled-duration illusion.

When a task requires subjects to estimate the temporal extent of a given interval, filling that interval with nontemporal stimuli (e.g., auditory, visual) will produce a change in duration judgments. The modification of duration judgments is not of the same magnitude for all levels of added stimulus complexity. If two values of a nontemporal stimulus dimension are paired orthogonally to one or more levels of duration, systematic perceptual distortions seem to occur. The higher value on the nontemporal dimension will produce longer apparent duration at each temporal interval. Warm and McCray (1969) found that an interval filled with frequent words seems to appear longer than an interval filled with less frequent words. Thomas and Cantor (1975) demonstrated that large stimuli tend to produce longer "perceived duration" than small stimuli. In the same vein, Canter and Thomas (1977) found that more complex stimuli result in longer "perceived duration" than less complex stimuli. These findings suggest that processing the nontemporal information influences the processing of temporal extent. Therefore, current explanations of the filled-duration illusion have placed little or no weight on processes which carry out direct timing of duration. Rather, the illusion effect has been characterized by appealing to the influence on temporal judgments of those mechanisms which analyze the auditory or visual information which fills the interval. 
The most prominent theoretical explanations of the filled-duration illusion rely either on stimulus encoding processes (Thomas \& Weaver, 1975) or memory storage mechanisms (Ornstein, 1969). While these theories stress different processes to characterize the illusion, they share an important underlying assumption. They assume the illusion phenomenon to be a function of properties present in each nominal stimulus event. The present experiments were designed to test this assumption.

The encoding model (Thomas \& Weaver, 1975) proposes two mechanisms, one which analyzes the nontemporal information and a second that times the duration. The filled-duration illusion results from an interaction of these mechanisms. It is assumed that attention cannot be allotted efficiently to both mechanisms when an interval is filled. The more nontemporal information in the stimulus, the more attention it demands, resulting in less attentional allotment to the temporal analysis. Within this framework, the direct time estimator or timekeeper has low attentional priority. Since most of the observer's attentional capacity is used to encode the nontemporal stimulus information, the output of the timekeeper is low in reliability or made unavailable (Thomas \& Brown, 1974; Thomas \& Weaver, 1975). Thus, the encoding model relies on the amount of real time a stimulus is shown to determine whether or not an illusion will occur. It is the absolute time that it takes to encode nontemporal information that is the critical variable. If the nontemporal aspects of the stimulus are encoded before the experimental interval ends, then more attention will be allocated to the timekeeper. Nontemporal information will be increasingly ignored as processing approaches completion. Thus, as durations lengthen, the illusion should be eliminated. Cantor and Thomas (1977) found that, at presentation durations of 30 and $70 \mathrm{msec}$, nontemporal stimulus variables produced the duration illusion. However, when they presented the same stimuli for 500 and $600 \mathrm{msec}$ (long past the time it would take to encode their stimulus items), they found no duration illusion. The resulting null effect at longer durations was taken as evidence that the illusion resided in the nontemporal stimulus processing stage. Encoding time and temporal discriminability were confounded in this procedure. When the intervals between experimental durations were long, the durations may have been very discriminable, and when the intervals were short, the durations may not have been very discriminable.

Ornstein's (1969) storage size model is distinguished from the encoding model by the assumption that there is no timekeeper or internal clock operating when filled intervals are judged. According to the model, duration judgments are always made by examining the load placed on memory by the nontemporal stimulus. Thus, temporal judgments are the sole result of nontemporal events. For example, a complex stimulus item will require a large amount of "mnemonic space" to be represented, while a less complex stimulus will be stored in less "mnemonic space." According to the storage size theory, perceived duration is a linear function of memory load, and temporal discriminability plays no role in the filled-duration illusion. Research done in this tradition (Block, 1974; Warm \& McGray, 1969) reflects this belief. In the typical paradigm, subjects are shown filled intervals of the same real-time durations and then asked to make a judgment that indicates which interval is long. er. Although this method is fine for demonstrating the systematic effects of nontemporal stimulus analysis on duration perception, it precludes any assessment of an interaction between temporal discrimination and the illusion.

The encoding and storage models both explain the illusion by appealing to the absolute characteristics of the presented stimulus on each trial, not to characteristics of the entire stimulus set (both temporal and nontemporal). Thus, if the level on a dimension (e.g., size, stimulus complexity, word frequency) which produces the illusion is held constant within an experimental session, the illusion should persist. Conversely, if the illusion is produced by perceptual or response strategies which are generated by properties of the stimulus set, then the filled-duration illusion should disappear when levels of a dimension are held constant across experimental sessions. The information processing strategy used to produce the illusion might be due, in part, to the correlation between the temporal and nontemporal dimensions. For example, there may be a strong tendency on the part of an ob. server to call a duration short when filled with a small object and long when filled with a large object. Since small objects can only be small relative to a large object, and large objects can only be large relative to a small object, the illusion should disappear when the information used to generate the strategy is no longer present.

The present investigation used two methods to address the possibility that a strategy lies at the root of the filled-duration illusion. The experiments manipulated the temporal and/or nontemporal context in which duration judgments are made.

\section{EXPERIMENT 1}

The influences of nontemporal set on the filledduration illusion are investigated by looking at changes in duration judgments as a function of pattern size. The size variable is studied under conditions where size is varied within a single group vs. those conditions where size is varied across experimental groups. Thus, some subjects are shown both large and small patterns at three exposure durations and asked to estimate temporal extent, while other subjects are 
shown only large or only small stimuli and asked to estimate temporal extent.

Temporal set influences are studied by varying the discriminability of the intervals presented to subjects. Some subjects see three durations separated by $15 \mathrm{msec}$, while other subjects see three durations separated by $70 \mathrm{msec}$. The goal of this procedure is to see what, if any, change temporal discrimination produces in the filled-duration illusion.

\section{Method}

Subjects. The subjects were graduate and undergraduate student volunteers at the University of California, Berkeley. They knew nothing of the experimental manipulation prior to participation. Thirty-two subjects participated, eight in each of four groups.

Stimuli. Two simple patterns were constructed, each including the same number of angles, same area, and same goodness. Goodness was defined according to Garner's (1974) formulation of rotation and reflection subsets. All stimuli had a rotation and reflection value of 4 . Figures were white patterns outlined in black and placed on a white background. Each figure was represented in two sizes, the smaller being 4/9 the size of the larger. The large figure when placed in the tachistoscope subtended approximately $4.0^{\circ}$ of the visual angle, while the small figure subtended approximately $2.5^{\circ}$. Two large figures and two small figures comprised the stimulus set (see Figure 1).

Design and Procedures. The subjects were shown the large and small figures either as a within-subjects or as a between-subjects variable. That is, half the subjects were shown the same sized figure every trial (constant groups), and half were shown the large and small stimuli randomized throughout the experiment (mixed groups). Eight subjects in the constant group saw a large-sized figure and eight saw a small-sized figure. Furthermore, four subjects in the constant-large condition and four subjects in the constantsmall condition were presented the stimuli at one set of intervals, while the remainder were shown the stimuli at a second set of intervals. The two intervals were manipulated by increasing or decreasing the temporal range. In the mixed conditions, one group saw the stimuli at one set of intervals, and the other saw them at the second set of intervals.

The two interval sets contained three time durations, either 15 , 30 , and $45 \mathrm{msec}$, called Set $I$. or 15,85 , and $155 \mathrm{msec}$, called Set II. Durations were chosen in the first set to have intervals separated by enough time for one to see the difference in the durations, but with maximal difficulty. The durations in the second set were chosen because they were readily discriminable, but not to the extent of producing perfect performance.

The design, then, had three levels of duration for each subject (short. medium, or long), two levels of interval differences between subjects (15 msec in Set I or $70 \mathrm{msec}$ in Set II), two types of pattern presentation between subjects (constant or mixed), and two sizes of stimuli (large or small) manipulated as a between or within subjects variable. The groups were Set I mixed, Set I constant, Set II mixed, and Set II constant. The constant groups could be broken down further into those presented large stimulus figures and those presented small stimulus figures.

Stimuli were presented one figure per trial on an Iconix threefield tachistoscope. The illumination was kept constant. Each trial first contained a 500-msec "warning" flash telling a subject that the trial had begun. Five hundred milliseconds after termination of the warning flash, a pattern flash was given for a duration corresponding to the particular condition and trial. A dark period of 500 msec followed the pattern and was ended by the advent of a display with the words SHORT, MEDIUM, and LONG written on it. Subjects were told to respond verbally when they saw this display and, when they had finished responding, to press a panel switch on a table in front of them. This switch turned off the final display

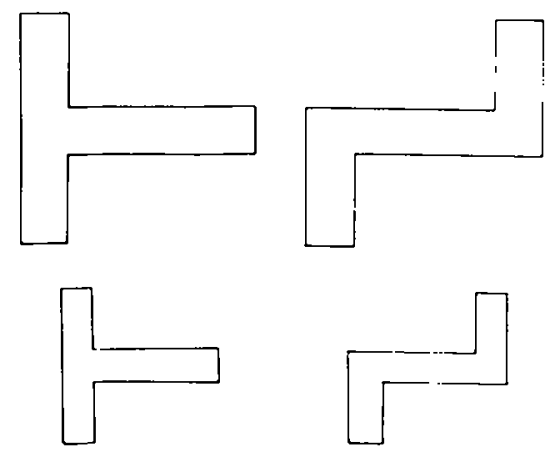

Figure 1. Stimulus set for Experiments 1 and 2.

light and reset the equipment for a new trial. The subjects were informed of the function of the switch.

Before the experimental trials began, all subjects were given 30 practice trials. During these trials, a rectangular pattern was presented. The height of this pattern was the average between the two stimulus size heights, and the width corresponded to the width between the interior lines of the small sized stimuli, i.e., 1/5 the total stimulus width.

On each of the practice trials, the subjects were told they were correct if that was the case or given the correct answer if they were incorrect. Feedback was given only during the initial $\mathbf{3 0}$ practice trials and at no other time during the experiment. This set of practice trials served to get the subjects accustomed to making duration decisions. Also, this period calibrated the subject's judgments to the durations used in any given condition.

Following the practice, 12 filler trials were presented to introduce the subjects to the target stimuli. The filler trials were not included in the analysis. A total of 96 experimental trials completed a session. After 48 trials, the subjects were given a short break. After the break, a second set of 12 fillers was presented. followed by the 48 remaining experimental trials.

Stimuli were presented in 10 blocks of 12 , including 9 experimental trials and 24 filler trials. For the mixed groups, each block contained three presentations of each stimulus. For example, one presentation of stimulus " 1 , large" was at the short duration, one at the medium, and one at the long. Five such randomizations were produced, one filler and four experimental, which took the subject through half a session. Two block sequence randomizations were used, each subject experiencing both, but with half the subjects in each group seeing Block Sequence I first and half seeing Block Sequence II first. Subjects in the constant conditions were shown the same sequence of durations as the mixed groups but without the changing sizes.

The sequence of events was fully outlined for the subjects during instructions. The subjects were told they would be shown examples of short, medium, and long durations; that there would be a pattern in the duration; and that they would have to concentrate or they could miss the pattern. They were not told that size would change. The subjects were asked to make overt judgments by clearly saying, "short," "medium," or "long" when the final display appeared. Responses were recorded by the experimenters.

\section{Results}

To quantify the dependent variable, the values of 0 , 1 , and 2 were given to short, medium, and long responses, respectively (short $=0$, medium $=1$, long $=2$ ). A mean response value was then obtained for each subject for each duration. The initial analysis did not include an evaluation of the effect of the large and 
small variables. That is, responses to large and small stimuli in the mixed conditions were collapsed over each subject, and subjects who saw large and small stimuli in the constant conditions were not considered as separate groups. This procedure allowed an analysis of the general impact of the difference in presentation. It resulted in an analysis of the two levels of interval set (15- or 70-msec difference), the two levels of nontemporal stimulus presentation (mixed or constant), and the repeated measure of durations (short, medium, and long).

The results showed a clear effect of temporal set on duration judgments. There was a large main effect of duration $[F(2,56)=30.70, p<.001]$ and a significant interaction of interval set with duration $[\mathrm{F}(2,56)$ $=178.38, \mathrm{p}<.001]$. As expected, when the interval differences were large, sensitivity (discrimination) to differences in duration increased. Each interval set showed a monotonically increasing function over durations, but the slope of Set II (70-msec differences) was much greater than that of Set I (15-msec differences). The one surprising result was a significant Mixed/Constant by Duration interaction $[F(2,56)=$ $7.27, \mathrm{p}<.01]$. Dunn's comparisons determined that the locus of the interaction was between the short and the medium durations $(F=14.92, p<.05)$. Three other Dunn's comparisons demonstrated no reliable differences between the mixed and constant groups.

The effects of large and small were analyzed separately in the constant and mixed conditions, since size was a within-subjects variable in one condition and a between-subjects variable in the other. The constant groups contained two levels of size, two levels of interval set, and three levels of presentation duration.

The role of nontemporal context in the illusion became apparent when the size factor was analyzed. The

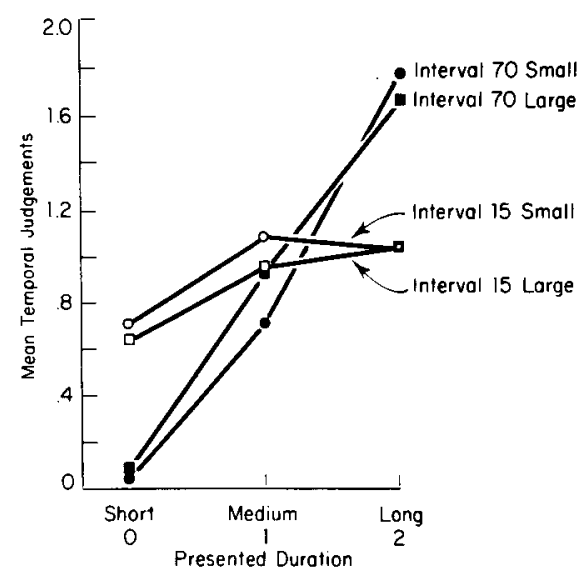

Figure 2. Mean temporal judgment at the three levels of presented duration for the 15 -msec-interval difference $(15,30,45 \mathrm{msec})$ vs. the 70 -msec-interval difference $(15,85,155 \mathrm{msec})$ with large vs. small stimuli as a between-subjects variable.

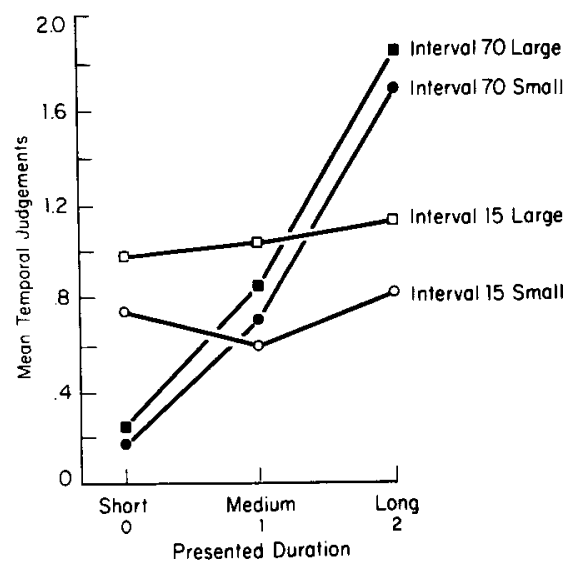

Figure 3. Mean temporal judgment at the three levels of presented duration for the 15-msec-interval difference $(15,30,45 \mathrm{msec})$ vs. the 70-msec-interval difference $(15,85,155 \mathrm{msec})$ with large vs. small stimuli as a within-subjects variable.

constant analysis resulted in a large main effect of duration $[F(2,24)=181.38, p<.001]$ and a significant Interval Set by Duration interaction $[F(2,24)$ $=78.26, \mathrm{p}<.001]$. No other effects were significant. Figure 2 depicts these results. There was nothing indicating the presence of a size effect. If anything, Figure 2 shows that at Interval Set I the trend was reversed.

The analysis for the mixed condition was essentially the same as for the constant condition, with the exception that size became a within-subjects variable. In line with the above results, a large main effect of duration was obtained $[F(2,28)=144.54, p<.001]$, as well as a large Interval Set by Duration interaction $[F(2,28)=105.95, p<.001]$. The magnitude of this effect can be viewed in Figure 3. Two other significant effects resulted, an effect of size $[F(1,14)=33.73$, $p$ $<.001]$ and a Size by Interval Set interaction $[F(1,14)$ $=6.63, \mathrm{p}<.05]$. Since size and interval were the main variables of concern, and since the predictions were based on the results from these manipulations, planned comparisons were run on the size and duration effects for the two different interval sets. When the interval difference was $\mathbf{1 5} \mathbf{~ m s e c}$, the main effect of duration vanished. Although the slope tended upward, the differences between durations were not significant. More importantly, the size effect was evident in a large main effect of size $[F(1,7)=26.00$, $p<.01]$. Size did not interact with duration $(F<1)$. The nearly parallel lines in Figure 3 depict this finding. Finally, it should be noted that all but one subject in the mixed condition had a greater mean response value to large stimuli than to small stimuli. The one aberrant subject showed no effect of size at all.

The analysis of Interval Set II in the mixed condition revealed that the overall main effect of duration 
was caused almost entirely by the 70 -msec interval difference. The effect of size was again found $[F(1,7)$ $=9.94, p<.025]$, but not to the degree of the Interval Set I group. Although the interaction was not significant, Figure 3 seemed to suggest an increasing difference between large and small stimuli as a function of longer durations in the 70-msec condition. Dunn's comparisons were performed on the size effect, and at the short and medium presented durations, the size effect was not significant. However, the difference between large and small at the long duration was significant $[F(1,7)=10.29, \mathrm{p}<.05]$. The size effect did occur when intervals were separated by $70-\mathrm{msec}$ differences, but it was highly attenuated.

\section{Discussion}

The results of Experiment 1 show that the environment in which judgments are made (provided by both the temporal and nontemporal contexts) will directly affect the presence or absence of the filled-duration illusion. Both of the prevailing theoretical perspectives fail to predict such findings.

Since the encoding and storage models base their ideas on absolute attributes of each stimulus event, the null size effects in the constant conditions would not be expected by theorists holding either position. A large stimulus item should take longer to process or require more storage space whether presented alone or in context with small stimuli.

The null effects in the constant conditions are more compelling when considered in conjunction with the effects of the mixed conditions. When size is varied within a session, a large effect is found when only $15 \mathrm{msec}$ separate the durations, while the effect is highly attenuated when there are 70 -msec differences. The data indicate that the size effect increases as duration uncertainty increases.

The storage model has the greatest difficulty with these results. According to Ornstein (1969), temporal discrimination is not a contributing factor in the filled-duration illusion. The current data show the illusion to be strongly influenced by temporal discriminability. These results do not rule out the possibility that memory mechanisms are a factor in the illusion, but the pattern in the data suggests that a memory storage model would have to be expanded to include the role of a timer.

The encoding model does predict the attenuation of the size effect as durations are lengthened. The model claims that the less time an item takes to encode, the more attention can be allotted to a timing mechanism. A subject will then rely more on this mechanism to make duration judgments. Thus, at longer durations, nontemporal information should exert little effect on the timekeeper.

A second study was conducted to test this assumption of the encoding model. Experiment 2 was de- signed to combine 15-msec interval differences with longer temporal intervals. The encoding model predicts little or no effect of size at these longer durations. The present hypothesis is not only that a size effect will result, but that it will approach the magnitude of the size effect of the 15 -msec interval difference condition of the first experiment.

\section{EXPERIMENT 2}

\section{Method}

Subjects. Eight subjects were drawn from the undergraduate population at Berkeley. All subjects were run as a mixed group.

Procedure. The same stimulus set was used, and the procedure was essentially the same as in Experiment 1, with the following exception. The durations were changed to 155,170 , and $185 \mathrm{msec}$. The values of the durations were longer than the durations used in Experiment 1, while the interval difference remained the same as Set $I$ in the first experiment.

\section{Results and Discussion}

Keeping the absolute durations long but reducing the difference between the durations resulted in the data that appear in Figure 4. It is clear from an inspection of the figure that this manipulation produced a reliable size effect $[F(1,7)=44.75, p<$ $.001]$. The subjects were also able to discriminate the short, medium, and long durations. This ability was evidenced by a significant main effect of duration $[F(2,14)=27.67, p<.001]$. Stimulus size did not differentially influence the ability to discriminate the three durations, since the size and duration factors did not interact significantly.

A comparison of Figures 3 and 4 suggests that the size illusion depicted in Figure 4 is more like the short-interval illusion than the long-interval illusion in Experiment 1. A test of these differences revealed that. the long-duration, short-interval illusion (Figure 4) was no different from the Experiment 1 Set I (15-msec) mixed illusion (Figure 3). The Experiment 1 Set II (70-msec) mixed size illusion did, how-

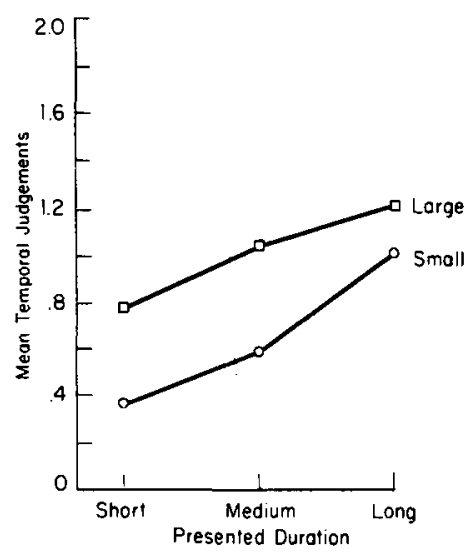

Figure 4. Mean temporal judgment at the three tevels of presented duration $(155,170,185 \mathrm{msec})$ with 15 -msec duration intervals for large and small stimuli. 
ever, differ from the Experiment 2 size illusion $[F(1,14)=11.68, p<.01]$.

The results from Experiment 2 clearly reveal that the absolute amount of real time available to encode the nontemporal stimulus is not the prime determiner of the filled-duration illusion. These data show a robust size illusion when the shortest presented duration is equal to the longest duration of the $70-\mathrm{msec}$ interval set in the first experiment.

Experiment 2, in combination with Experiment 1, makes more apparent the role of temporal discrimination ability in the filled-duration illusion. These results show that as temporal discrimination becomes more difficult, as measured by the decrease in slope, the filled-duration illusion increases in magnitude. This conclusion must be tempered by the fact that the current procedure forced subjects to use three categories in making their judgments. It may be that, given a continuous or functionally infinite response scale, the relationship between discriminability and the magnitude of the illusion would change.

An important aspect of the results in both experiments is that the ability to see changes in real temporal extent, independent of the information which fills the interval, is never completely lost. As long as the difficulty of the temporal discrimination is kept constant (Experiment 2 and the 15-msec difference condition in Experiment 1), the results suggest that the role of the timekeeper in the illusion will be about the same.

\section{GENERAL DISCUSSION}

Current theorizing concerning the filled-duration illusion has assumed the illusion to be a function of one or another absolute parameter of each stimulus event. The findings presented here have called this assumption into question. Three important conclusions emerge from these data. First, nontemporal properties of each stimulus in isolation are insufficient to produce an illusion. Second, the illusion can be produced at relatively short and at relatively long durations. Finally, the data show that temporal discriminability and the filled-duration illusion bear interesting relationships to each other.

Results from Experiment 1 demonstrate that pattern size can only produce a duration illusion if observers are aware that different sizes exist in the stimulus set. These findings reveal that there is nothing inherent in the size of any given stimulus item which would make an information processor overestimate or underestimate a duration. The tendency to change duration judgments as a function of pattern size is a result of the stimulus set, not the characteristics of any given pattern.

Taken together, both experiments show the filledduration illusion to be insensitive to large changes in the amount of real time provided for nontemporal stimulus encoding. The magnitude of the illusion effect is approximately the same at durations of 15 , 30 , and $45 \mathrm{msec}$ as it is at 155,170 , and $185 \mathrm{msec}$. Therefore, real time properties of each stimulus presentation do not cause the illusion. Alternatively, the range of durations in the stimulus set certainly plays a role in the illusion.

The current data have shown that the ability to see the difference between long and short durations is not completely lost even in the presence of a robust illusion. This finding suggests that a time-keeping process and processes which analyze nontemporal information are both contributing factors to the illusion. When discrimination between durations is made easier, the process of timekeeping is obvious, while the nontemporal stimulus set seems to decrease in its influence on temporal judgments. As noted above, this conclusion cannot be definitively stated, since a continuous response scale was not used in the present investigation.

By showing the filled-duration illusion to be largely a function of context, the current data have created a theoretical void. The theory which fills this void could be based on strategies that are generated by a perceptual or response system. It could be the case that the perceptual system, when trying to analyze brief durations, applies a correction for the size or complexity of the information that fills the duration. This correction process may make differential adjustments to large and small stimuli when the durations are close in temporal extent. The nature of the correction may cause large stimuli to appear as if they had been exposed longer.

It is equally plausible that when durations are hard or impossible to distinguish, a response bias is induced into the information processing system. This bias may cause temporal processing to be ignored and temporal responses to be replaced by responses based on the nontemporal dimension. For example, when a pattern is large, the response system would, with some high probability, produce the output "long" for the duration estimation. Similarly, when a pattern is small, the system, with equally high probability, would respond "short."

The present data make these theoretical conjectures reasonable. The findings cannot, however, differentiate between them.

\section{REFERENCES}

Allan, L. G., \& Kristofferson, A. B. Psychophysical theories of duration discrimination. Perception \& Psychophysics, 1974, 16, 26-34.

Avant, L. L., Lyman, P. J., \& Antes, T. R. Effects of stimulus familiarity upon judged visual duration. Perception \& Psychophysics, 1975, 17, 253-262.

BLOCK, R. A. Memory and the experience of duration in retrospect. Memony \& Cognition. 1974, 2, 153-160. 
Buffardi, L. Factors affecting the filled duration illusion in the auditory, tactual, and visual modalities. Perception \& Psychophysics, 1971, 10, 292-294.

Cantor, N. E., \& Thomas, E. A. C. The control of attention in the processing of temporal and spatial information in complex visual patterns. Journal of Experimental Psychology: Human Perception \& Performance, 1977, 3, 243-250.

GARNER, W. R. The processing of information and structure. Potomac, Md: Erlbaum, 1974.

ORNSTEIn, R. E. On the experience of time. Middlesex, England: Penguin Books, 1969.

Thomas, E. A. C., \& Brown, I. Time perception and the filled duration illusion. Perception \& Psychophysics, 1974, 16, 449-458.
Thomas, E. A. C., \& Cantor, N. E. On the duality of simultaneous time and size perception. Perception \& Psychophysics, 1975, 18, 44-48.

Thomas, E. A. C., \& Weaver, W. B. Cognitive processing and time perception. Perception \& Psychophysics, 1975, 17, 363-367.

WARM, J. S., \& MCCRAY, R. E. Influence of word frequency and length on the apparent duration of tachistoscopic presentations. Journal of Experimental Psychology, 1969, 79, 56-58.

(Received for publication March 27, 1978; revision accepted January 26, 1979.) 